\title{
How sound is the evidence that thrombolysis increases the risk of cardiac rupture?
}

\author{
David R Massel
}

Rupture of the free wall of the heart is a catastrophic and usually lethal event. It occurs in up to $10 \%$ of patients dying in hospital of acute myocardial infarction. While there is unequivocal evidence for the long-term benefit of thrombolytic therapy in patients with suspected myocardial infarction, in many streptokinase trials there were more deaths during the first 24-36 hours in treated patients than in controls. ${ }^{1}$ It has been suggested that thrombolytic therapy increases the risk of cardiac rupture as a consequence of extensive myocardial haemorrhage and that this effect may partly explain this early hazard. The issue may be addressed from the following perspectives:

- Is there evidence from animal studies that thrombolysis promotes myocardial haemorrhage?

- Does myocardial haemorrhage occur and increase the risk of rupture in humans?

- Is there evidence from randomised trials that the use of thrombolytic therapy increases the risk of cardiac rupture?

- What does this mean in the overall context of risk versus benefit when patients are selected for coronary thrombolysis?

\section{Myocardial haemorrhage: animal evidence}

A review of animal studies indicates that early reperfusion leads to salvage of myocardium but that it may also result in morphological differences, including myocardial haemorrhage, in the necrotic myocardium as compared with non-reperfused infarcts. ${ }^{2-14}$ The potential adverse consequences of myocardial haemorrhage include extension of ischaemic injury, delayed healing, and functional myocardial abnormalities. Haemorrhagic necrosis is directly related to the duration of occlusion and the extent of damage to the microvasculature. ${ }^{2-5}$ It occurs within the ischaemic zone where vascular death has followed myocardial cell death-that is, in regions with irreversible injury. ${ }^{6}$ Moreover, myocardial haemorrhage is likely to be related to reperfusion and not to thrombolysis itself. The addition of streptokinase does not lead to a greater degree of haemorrhage than that experienced with reperfusion alone. ${ }^{78}$

Studies suggesting an extension of ischaemic injury caused by myocardial haem- orrhage relied on serial serum creatine kinase (CK) concentrations to estimate infarct size. ${ }^{9}$ It has been shown, however, that reperfusion enhances the release of $\mathrm{CK}$ per gram of infarcted myocardium. ${ }^{15}{ }^{16}$ Therefore, serial CK determinations are an inaccurate means of predicting extent of myocardial infarction after reperfusion. ${ }^{15} 16$

The effects of thrombolysis on the healing process within infarcted myocardium are controversial. ${ }^{911}$ Impaired healing has been suggested because reperfusion leads to an altered early granulocyte response. ${ }^{10}$ However, coronary reperfusion after myocardial infarction alters the pattern of injury and the cellular response to the evolving myocardial infarction, so that classic criteria for infarct age may not apply. ${ }^{17}$ For infarcts of the same clinical age, those receiving reperfusion therapy were judged to have older histological ages. ${ }^{17}$ Reperfusion does not seem to have a detrimental effect on late collagen formation. ${ }^{10} 1213$

There is no evidence that infarct size is increased after reperfusion and myocardial haemorrhage. ${ }^{2578101213}$ With short durations of occlusion ( $<120$ minutes ) infarct size was smaller than the potential area at risk. ${ }^{17}$ This suggests that any deleterious effect of myocardial haemorrhage would be less important than the benefit of reperfusion. With longer periods of occlusion, infarcts were transmural and infarct size was not increased beyond that predicted. ${ }^{17}$ There are no data on the effect of myocardial haemorrhage on longterm left ventricular function.

\section{Myocardial haemorrhage: postmortem studies in humans}

Myocardial haemorrhage has been observed in humans after cardiac surgery, coronary angioplasty, and thrombolytic therapy. Though cardiac rupture has been found in association with myocardial haemorrhage, it remains unclear whether the risk is increased after thombolysis or whether any observed association is the result of case selection and reporting.

A postmortem study by Cowan et al suggested that early myocardial haemorrhage may increase the risk of ventricular rupture.$^{17}$ Forty three cases of death after reperfusion therapy were collected prospectively from 36 hospitals in Washington state and compared 
with matched controls (no reperfusion therapy) derived from the files of the principal investigator and two local hospitals. Eight of the haemorrhagic infarcts after reperfusion therapy showed ventricular rupture and four of the infarcts were less than 24 hours old. In contrast, 890 files had to be reviewed to match four controls with cardiac rupture within 24 hours of myocardial infarction.

Gertz et al examined the hearts of 61 patients with a first fatal myocardial infarction: 23 received recombinant tissue-type plasminogen activator and 38 did not. ${ }^{18}$ Patients died a median of 2.0 and 4.2 days respectively from symptom onset. The frequency of cardiac rupture was lower among those receiving thrombolytic therapy $(22 \% v$ $47 \%, p=0.045)$. Similarly, the frequency of rupture among the patients who died within 72 hours of symptom onset was also lower among those patients who received recombinant tissue-type plasminogen activator than among those who did not $(20 \% v 64 \%, \mathrm{p}=$ $0 \cdot 02)$.

Gertz et al also examined 52 hearts of patients who had received recombinant tissue-type plasminogen activator for evolving myocardial infarction as part of the Thrombolysis in Myocardial Infarction (TIMI) study and who died at a median of $2 \cdot 7$ days from symptom onset. ${ }^{19}$ Myocardial necrosis was confirmed histologically in 43 patients: 23 infarcts were grossly haemorrhagic and 20 were non-haemorrhagic. Haemorrhage was confined to areas of necrosis in all cases. The frequency of cardiac rupture was similar in the haemorrhagic (26\%) and non-haemorrhagic (25\%) infarctions.

Kleiman et al analysed the cause of death in 63 patients who died within the first 18 hours of enrolment in the TIMI Phase II study. ${ }^{20}$ Ventricular rupture was deemed to be the cause of death when there was direct evidence from visualisation of the heart or when sudden death occurred in the setting of continuously monitored and normal blood pressure and rhythm. Pump failure was responsible for $62 \%$ of early deaths, cardiac rupture for $16 \%$, arrhythmias for $13 \%$, and complications of therapy for $9 \%$. In the subgroup of patients without initial hemodynamic compromise, rupture accounted for a larger proportion of deaths but was still less common than pump failure. It was more likely to be diagnosed in patients who had necropsy or surgical exploration of the chest, suggesting that clinical suspicion of rupture may have led to more frequent necropsies. The effect of early and deferred $\beta$ blockade was assessed within a subgroup of the TIMI II trial. The small number of deaths in each category precluded the demonstration of any effect of $\beta$ blocker therapy on rupture. Previously, an effect of $\beta$ blockers on early cardiac rupture was proposed after retrospective review of the First International Study of Infarct Survival (ISIS-1), a randomised trial to assess the effect of early $\beta$ blockade on survival after myocardial infarction. ${ }^{21}$ Almost all of the mortality reduction associated with the use of atenolol occurred on days 0 and 1 . Significantly fewer deaths ascribed to cardiac rupture (confirmed by necropsy) or to electromechanical dissociation occurred in the atenolol group than in the controls (20 v $54,2 \mathrm{p}<0.02)$.

A retrospective review of the 1386 in-hospital deaths in the Gruppo Italiano per lo Studio della Streptochinasi nell'Infarcto Miocardio (GISSI) trial showed that the most frequent cause of death was cardiac failure $(52 \%)$, which was significantly less common in treated patients than in controls. ${ }^{22}$ No differences between treatment groups were observed for other causes of death including electromechanical dissociation, sudden cardiac death, and deaths from extracardiac causes. While cardiac rupture accounted for $9 \cdot 1 \%$ of total deaths, it was overrepresented (58\%) among the $16 \%$ of patients who died and underwent necropsy in this unblinded trial. ${ }^{23}$ Diagnostic suspicion may have resulted in a necropsy being performed more often in cases of rupture.

\section{Cardiac rupture: evidence from randomised trials}

On the basis of a review of the causes of death in two placebo controlled trials, Vasilomanolakis et al suggested that streptokinase predisposes to cardiac rupture. The differences observed (24\% streptokinase, $16 \%$ placebo), however, may have been due to chance $(p=0.54) .{ }^{24}$ An overview of 33 randomised trials of thrombolysis performed before 1985 did not find an excess rate of cardiac rupture among those treated with a fibrinolytic agent for myocardial infarction. ${ }^{25}$ The reliability of these analyses is limited, however, because not all studies reported deaths, and necropsy findings or the rate of necropsy was low. In addition, the thrombolytic dose protocols and concurrent therapies in these trials were not typical of current practice, so any generalisations are tenuous.

In the Intravenous Streptokinase in Acute Myocardial Infarction (ISAM) study $1 \cdot 2 \%$ of those treated with streptokinase had ventricular septal rupture or clinically suspected rupture of the free wall within 21 days of randomisation compared with $1.5 \%$ of controls. ${ }^{26}$ Of cardiac deaths, rupture (septal and free wall) was the cause among $20.4 \%$ $(10 / 49)$ of streptokinase treated patients and $21.0 \%(13 / 62)$ of controls. Rupture caused only two (1/17 control, $1 / 12$ streptokinase) of the 29 deaths occurring within 14 days in the Western Washington study. ${ }^{27}$

In the ISIS-2 trial cardiac rupture was reported in $0.9 \%$ of both the streptokinase and placebo groups. ${ }^{28}$ The combination of streptokinase and aspirin was associated with a $0.2 \%$ absolute reduction in the rate of cardiac rupture $(0.9 \% v 0.7 \%)$. In addition, cardiac arrests from causes other than ventricular fibrillation (possibly electromechanical dissociation) were reduced by streptokinase and the combination of streptokinase and aspirin. Though there were 
more deaths in the streptokinase group within the first 24-36 hours the rates of cardiac rupture seemed to be similar. ${ }^{1}$ In the GISSI trial there was no difference in the rate of cardiac rupture $(1.0 \%$ streptokinase, $1.1 \%$ controls). ${ }^{23}$

Therefore, these studies do not provide evidence that thrombolysis increases the overall risk of cardiac rupture. Honan et al examined the relation between the risk of cardiac rupture and the timing of thrombolytic therapy for myocardial infarction by performing a meta-analysis of placebo controlled trials where cardiac rupture was reported and the necropsy rate was $>50 \% .^{29}$ The limitations of this meta-analysis have been discussed elsewhere and caution urged in the interpretation of the results. ${ }^{30}$ Overall, rates of cardiac rupture among those receiving streptokinase $(29 / 831)$ and controls (29/807) were similar. However, Honan et al suggested that the risk of rupture was biphasic and directly related to the timing of thrombolytic therapy in relation to the onset of symptoms: early treatment $(<7 \mathrm{~h})$ decreases the risk and late treatment $(>17 \mathrm{~h})$ increases the risk. The results from the GISSI trial were taken as corroborating evidence despite the low rate of necropsy $(16 \%)$ and the unblinded nature of the trial.

\section{Clinical context}

There is unequivocal evidence supporting the benefit of thrombolytic therapy given up to six hours from the onset of myocardial infarction. ${ }^{1}$ There is considerable evidence that patients who start treatment at 6-24 hours may still benefit significantly though the size of benefit seems to diminish the later the onset of therapy. ${ }^{31}$ Because of the possibility of hazard associated with the use of thrombolysis, particularly if administered late, perhaps more emphasis should be given to the relative contraindications when the risk/benefit ratio in an individual is formulated.

Pump failure, not cardiac rupture, continues to be the predominant cause of early and late death after thrombolysis and strategies to limit infarct size are certainly worth investigating. Examination of the causes of death in continuing large clinical trials, especially those such as GISSI-3 and ISIS-4, may provide further insight into this problem.

\section{Conclusions}

Myocardial haemorrhage occurs as a consequence of reperfusion, not thrombolytic therapy in itself, and is limited to areas of irreversible injury. Although cardiac rupture is associated with myocardial haemorrhage it remains unclear whether this association is causal. There is no strong evidence to suggest that coronary thrombolysis increases the overall risk of cardiac rupture or explains the early risk. Pump failure continues to be the predominant cause of death in patients receiving thrombolytic therapy. Nevertheless, the results of the study by Honan et al, suggesting an increased risk of rupture with delayed therapy, deserve further investigation. If nothing else, this study would compel researchers to investigate mechanisms of death after thrombolysis and to design adjunctive strategies to reduce myocardial damage and improve the prognosis of patients with myocardial infarction.

1 Yusuf S, Sleight P, Held P, McMahon S. Routine medical management of acute myocardial infarction. Lessons learned from overviews of recent randomized controlled trials. Circulation 1990;82(suppl II):117-34

2 McNamara JJ, Lacro RV, Yee M, Smith GT Hemorrhagic infarction and coronary reperfusion. $f$ Thorac Cardiovasc Surg 1981;81:498-501.

3 Cerra FB, Lajos TZ, Montes M, Siegel JH. Hemorrhagic infarction: A reperfusion injury following prolonged myocardial ischemic anoxia. Surgery 1975;78:95-104.

4 Higginson LAJ, White F, Heggtveit HA, et al. Determinants of myocardial haemorrhage after coronary reperfusion in the anesthetized dog. Circulation 1982; 65:62-9.

5 Fishbein MC, Y-Rit J, Lando U, et al. The relationship of vascular injury and myocardial haemorrhage to necrosis after reperfusion. Circulation 1980;62:1274-9.

6 Kloner RA, Rude RE, Carlson N, et al. Ultrastructura evidence of microvascular damage and myocardial cel injury after coronary artery occlusion: Which comes first? Circulation 1980;62:945-52.

7 Higginson LAJ, Sheldrick KR, Temple V, Beanlands DS Intracoronary streptokinase: Effects of reperfusion haemorrhage and regional myocardial blood flow in the anesthetized dog. 7 Cardiovasc Pharmacol 1987; 9:509-14.

8 Kloner RA, Alker KJ. The effect of streptokinase on intramyocardial haemorrhage, infarct size, and the noreflow phenomenon during coronary reperfusion. refiow phenomenon during
Circulation 1984;70:513-21.

9 Bresnahan GF, Roberts R, Shell WE, Ross J Jr, Sobel BE. Deleterious effects due to haemorrhage after myocardia reperfusion. Am $\mathcal{F}$ Cardiol 1974;33:82-6.

10 Roberts CS, Schoen FJ, Kloner RA. Effect of coronary reperfusion on myocardial haemorrhage and infarct healing. Am f Cardiol 1983;52:610-4.

11 Althaus V, Gurtner HP, Baur H, Hamburger S, Roos B. Consequences of myocardial reperfusion following tem porary coronary occlusion in pigs: Effects on morphologic, biochemical and haemodynamic findings. Eur $\mathscr{f}$ Clin Invest 1977;7:437-43.

12 Geft IL, Fishbein MC, Hashida J, et al. Effects of late coronary artery reperfusion after myocardial necrosis is complete. Am Heart $\mathcal{~} 1984 ; 107: 623-9$.

13 Karsch KR, Hofman M, Rentrop KP, Blanke H, Schape W. Thrombolysis in acute experimental myocardial infarction. $\mathcal{F}$ Am Coll Cardiol 1983;1:427-35.

14 Garcia-Dorado D, Theroux P, Solares J, et al. Determinants of haemorrhagic infarcts: Histologic observations from experiments involving coronary occlusion, coronary reperfusion, and reocclusion. $\mathrm{Am} \mathcal{F}$ occlusion, coronary repe

15 Jarmakani JM, Limbird L, Graham TC, Marks RA. Effect of reperfusion on myocardial infarct and the accuracy of of reperfusion on myocardial infarct and the accuracy of estimating infarct size from serum creatine pho

16 Vatner SF, Baig H, Manders WT, Maroko PR. Effects of coronary artery reperfusion on myocardial infarct size coronary artery reperfusion on myocardial infarct size calculated from creatine kinase. $\mathcal{F}$ Clin Invest 1978;61:1048-56.

17 Cowan MJ, Reichenbach D, Turner P, Thostenson C. Cellular response of the evolving myocardial infarction after therapeutic coronary artery reperfusion. Hum Pathol 1991;22:154-63.

18 Gertz SD, Kragel AH, Kalan JM, et al. Comparison of coronary and myocardial morphologic findings in patients with and without thrombolytic therapy during fatal first acute myocardial infarction. Am $\mathcal{f}$ Cardiol 1990;66:904-9.

19 Gertz SD, Kalan JM, Kragel AH, et al. Cardiac morphologic findings in patients with acute myocardial infarction treated with recombinant tissue plasminogen activator. Am 7 Cardiol 1990;65:953-61.

20 Kleiman NS, Terrin M, Mueller $\mathrm{H}$, et al. Mechanisms of early death despite thrombolytic therapy: Experience early death despite thrombolytic therapy: Experience II (TIMI II) study. 7 Am Coll Cardiol 1992;19:1129-35.

21 ISIS-1 (First International Study of Infarct Survival) Collaborative Group. Mechanisms of the early mortality reduction produced by beta-blockade started early in acute myocardial infarction: ISIS-1. Lancet 1988; i:921-3.

22 Mauri F, De Biase AM, Franzosi MG, et al. GISSI: Analasi delle cause di morte intraospedaliera. G Ital Cardiol 1987;17:37-44

23 Franzosi MG, Mauri F, Pampallona S, et al. The GISSI Study: further analysis. Circulation 1987;76:(suppl II):52-6. 
24 Vasilomanolakis EC, Famularo MA, Kozlowski J, et al. Cardiac rupture following intracoronary streptokinase. Cardiac rupture following intracoronary
Cathet Cardiovasc Diagn 1983;9:291-6.

25 Yusuf S, Collins R, Peto R, et al. Intravenous and intracoronary fibrinolytic therapy in acute myocardial infarction: Overview on mortality, re-infarction and side-effects from 33 randomized controlled trials. Eur Heart f 1985;6:556-85.

26 The ISAM Study Group. A prospective trial of intravenous streptokinase in acute myocardial infarction (ISAM). $N$ Engl F Med 1986;314:1465-71.

27 Kennedy JW, Martin GV, Davis KB, et al. The Western Washington intravenous streptokinase in acute myocardial infarction randomized trial. Circulation 1988,
77:345-52.

28 ISIS-2 (Second International Study of Infarct Survival) Collaborative Group. Randomised trial of intravenous streptokinase, oral aspirin, both, or neither among streptokinase, oral aspirin, both, or neither among
17187 cases of suspected acute myocardial infarction: 17187 cases of suspected acu
ISIS-2. Lancet 1988;ii:349-60.

29 Honan MB, Harrell FE, Reimer KA, et al. Cardiac rupture, mortality and the timing of thrombolytic therapy: a meta-analysis. $\mathcal{F} \mathrm{Am}$ Coll Cardiol 1990;16:359-67.

30 Massel D. Cardiac rupture and time to thrombolytic treatment. J Am Coll Cardiol 1991;17:1671-2.

31 White HD. Thrombolytic therapy for patients with myocardial infarction presenting after six hours. Lancet 1992;340:221-2. 